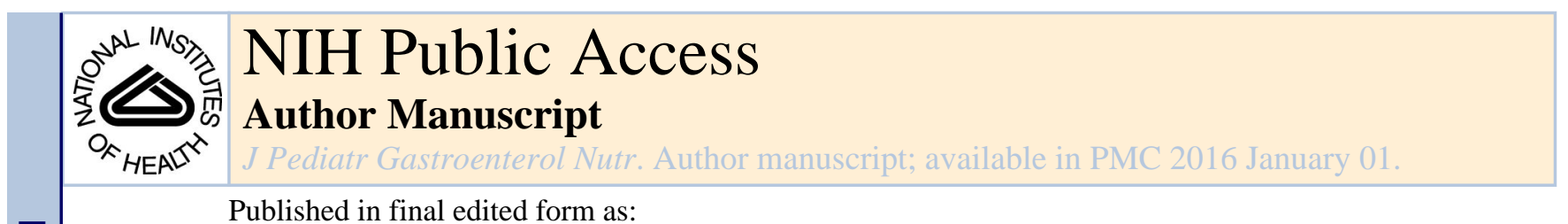

Published in final edited form as:

J Pediatr Gastroenterol Nutr. 2015 January ; 60(1): 18-22. doi:10.1097/MPG.0000000000000553.

\title{
Novel Risk Factors for Recurrent Clostridium difficile Infection in Children
}

\author{
Maribeth R. Nicholson, MD, MPH ${ }^{1}$, Isaac P. Thomsen, $\mathbf{M D}^{2}$, James C. Slaughter, $\mathrm{PhD}^{3}, \mathrm{C}$. \\ Buddy Creech, MD, $\mathbf{M P H}^{2}$, and Kathryn M. Edwards, $\mathbf{M D}^{2}$ \\ ${ }^{1}$ Division of Pediatric Gastroenterology, Hepatology, and Nutrition, Vanderbilt University School of \\ Medicine \\ ${ }^{2}$ Division of Pediatric Infectious Disease, Vanderbilt University School of Medicine \\ ${ }^{3}$ Department of Biostatistics, Vanderbilt University
}

\section{Abstract}

Objectives-Clostridium difficile, a common cause of antibiotic-associated diarrhea, has been reported to recur in high rates in adults. The rates and risk factors for recurrent Clostridium difficile infection (rCDI) in children have not been well established.

\begin{abstract}
Methods-We conducted a retrospective cohort study of 186 pediatric patients seen at a tertiary care referral center over a 5-year period diagnosed with a primary infection with Clostridium difficile. Children with recurrent disease, defined as return of symptoms of Clostridium difficile infection and positive testing $\$ 0$ days after the completion of therapy, were compared to children who did not experience an episode of recurrence.
\end{abstract}

Results-Of the 186 pediatric patients included in this study, 41 (22\%) experienced recurrent Clostridium difficile infection. On univariable analysis, factors significantly associated with recurrent Clostridium difficile infection included malignancy, recent hospitalization, recent surgery, antibiotic use, number of antibiotic exposures by class, acid blocker use, immunosuppressant use, and hospital acquired disease. On multivariable analysis, malignancy $(\mathrm{OR}=3.39,95 \% \mathrm{CI}=1.52-7.85)$, recent surgery $(\mathrm{OR}=2.40,95 \% \mathrm{CI}=1.05-5.52)$, and the number of antibiotic exposures by class $(\mathrm{OR}=1.33,95 \% \mathrm{CI}=1.01-1.75)$ were significantly associated with recurrent disease in children.

\footnotetext{
Address correspondence to: Maribeth R. Nicholson, MD, Division of Pediatric Gastroenterology, Hepatology, and Nutrition. Monroe Carell Jr. Children's Hospital at Vanderbilt 2200 Children's Way, Nashville TN 37232. Phone: (615)322-7449, Fax: (615)936-8128, maribeth.r.nicholson@ vanderbilt.edu.

Financial Disclosure: The authors have no financial relationships relevant to this article to disclose.

Author's Contributions:

Maribeth R. Nicholson: Dr. Nicholson conceptualized and designed the study, collected the data, performed the statistical analysis, drafted the initial manuscript, and approved the final manuscript as submitted.

Isaac P. Thomsen, C. Buddy Creech, and Kathryn M. Edwards assisted with study design, reviewed and revised the manuscript for important intellectual content, and approved the final manuscript as submitted.

James C. Slaughter assisted with study design, performed the statistical analysis, reviewed and revised the manuscript for important intellectual content, and approved the final manuscript as submitted.
} 
Conclusions-The rate of recurrent Clostridium difficile infection in children was $22 \%$.

Recurrence was significantly associated with the risk factors of malignancy, recent surgery, and the number of antibiotic exposures by class.

\section{Keywords}

Clostridium difficile; antibiotic-associated diarrhea; diarrhea; infectious colitis; hospital-acquired infection

\section{Introduction}

Clostridium difficile, a spore forming gram-positive bacillus, is the most frequent cause of antibiotic-associated diarrhea. ${ }^{1}$ Although children have not previously been known to be at high-risk for Clostridium difficile infection (CDI), recent studies have demonstrated that CDI is currently on the rise in children in both inpatient and outpatient settings. ${ }^{2,3}$ In the last ten years, the rate of pediatric hospitalization with CDI has nearly doubled. ${ }^{4}$

In adults the treatment of CDI is complicated by a very high rate of recurrent disease, with estimates of $20-30 \%$ of patients experiencing a recurrence, and multiple occurrences associated with increasing morbidity. ${ }^{5-7}$ Prior studies in adults have demonstrated that after a single episode of recurrence, 45 to $65 \%$ of patients will have repeated episodes of CDI that may continue over a period of years. ${ }^{8,} 6,{ }^{9}$ Recurrent CDI (rCDI) is often poorly responsive to treatment, requiring additional medications, longer courses of therapy, additional inhospital contact procedures, substantially increased medical costs, as well as increased risk of morbidity and mortality. In one study, the treatment of recurrent episodes of CDI required an average of 265 additional days/patient of vancomycin and 19.7 days/patient of metronidazole. ${ }^{8}$ The additional medical care and costs associated with rCDI are substantial.

Studies have begun to define important risk factors for rCDI in adults. A meta-analysis identified age greater than 65 years old, the use of concurrent antibiotics, and the use of gastric acid suppressants to increase the risk of rCDI in adults. ${ }^{10}$ Other studies have identified low serum anti-toxin antibody levels and hospital exposures as important risk factors for recurrence. ${ }^{11-13}$ Recent attempts have been made to create a clinical risk prediction model in adults to help determine the risk of recurrent disease at the time of the initial contact with a healthcare worker. ${ }^{14}$ There is a paucity of data, however, regarding risk factors for rCDI in children. While concurrent antibiotics and community-associated CDI were recently shown to be associated with an increased likelihood of rCDI in a pediatric population, ${ }^{15}$ a comprehensive assessment of host factors that govern rCDI risk is needed. The purpose of the current study is to identify independent risk factors for rCDI in children using rigorous statistical methods applied to a retrospective cohort from a large tertiary care children's hospital.

\section{Methods}

\section{Patient Selection}

With institutional review board exemption, a pediatric cohort was retrospectively compiled of 295 patients who had an episode of CDI based on positive laboratory testing at Monroe 
Carell Jr. Children's Hospital at Vanderbilt (MCJCHV) from January 1, 2007 through December 31, 2011, in both inpatient and outpatient settings. The episode of CDI was confirmed to be the primary infection, and not a recurrence, through review of the medical record. The outcome of interest was rCDI, defined as a recurrence of symptoms and positive testing for $C$. difficile occurring $\mathbf{5 0}$ days from the completion of the primary treatment for CDI.

During all but the last two months of the study period, laboratory testing for $C$. difficile consisted of an enzyme immunoassay for toxin (Meridian Bioscience Premier). In November 2011, DNA amplification (Illumigene $C$. difficile assay, ARUP laboratories) was begun.

Eligible patients were between the ages of 12 months to < 18 years with medically documented diarrhea and confirmatory $C$. difficile laboratory testing. The description of diarrhea needed to include $>1$ episode of stooling in a 24 hour period with stools described as "loose," "watery," or "unformed." Children less than 12 months of age were excluded from the study due to the known high rate of asymptomatic colonization in this demographic. ${ }^{16}$

Patients were excluded from the study if they were missing follow-up information after 60 days of completion of therapy; if they were not treated for primary CDI; if they died during the follow-up period; or if they were treated for an episode of rCDI without the presence of diarrhea and/or laboratory confirmation. Furthermore, patients were excluded if they were treated with an antibiotic known to be effective against $C$. difficile for a non-C.difficile indication during the follow-up period.

The type of CDI was classified per standard definitions as healthcare facility-onset, healthcare facility-associated (HO-HCFA); community-associated Clostridium difficile associated disease (CA-CDAD); community onset, healthcare facility-associated disease (CO-HCFA); and indeterminate. ${ }^{17}$

After inclusion and exclusion criteria were carefully considered, 186 subjects out of the 295 patients with CDI were included in the study. The final selection of the cohort is illustrated in Figure 1.

\section{Data Collection}

We collected demographic data for the 186 subjects in the cohort. In addition, we specifically collected patient comorbidities, hospitalizations, surgeries, and medication use. Hospitalizations and surgeries were identified 60 days prior to the onset of symptoms of CDI. Medication use was collected for 30 days prior to the onset of symptoms of CDI with a focus on antibiotic, immunosuppressant, and acid blocker use. In patients that received antibiotics during the 30 day period, the number of classes of antibiotics that were used during this time was also collected. Characteristics of the initial infection, including complications and treatment, were obtained. All data were collected from the comprehensive electronic medical record system at Vanderbilt, which includes all inpatient and outpatient 
encounters, as well as records sent to Vanderbilt from other non-Vanderbilt clinics and hospitals.

Data were managed using REDCap (REDCap software, Vanderbilt University) ${ }^{18}$ and analyzed using Stata (Stata Corp., College Station, TX) and R (R Core Team, Vienna, Austria).

\section{Data Analysis}

Patients with and without rCDI were compared using Pearson's Chi-squared test for categorical data and Wilcoxon rank-sum test for continuous data.

We pre-specified 11 major predictors that we hypothesized would be related to the risk of recurrent disease in children due to their presence as risk factors for either primary disease in children or adults or recurrent disease in adults. ${ }^{10,19-21}$ These predictors included the use of the following medications in the 30 days prior to symptom onset: antibiotics (both as a dichotomous variable and the number of classes), acid blockers (including $\mathrm{H} 2$ receptor antagonists and/or proton pump inhibitors), proton pump inhibitors (PPIs) alone, and immunosuppressants. We predicted that patients with HO-HCFA disease or a hospitalization or surgery within a 60-day period prior to symptom onset would be at higher risk of rCDI as well as those with a diagnosis of malignancy or the presence of a feeding tube. Finally, we hypothesized that the absence of fever at the time of symptom onset would increase the risk of recurrent disease. This hypothesis was based on the association of low serum antibody response to toxin A and increased risk of rCDI. ${ }^{13}$ We hypothesized that those patients with a less vigorous immune response, demonstrated by the absence of fever, would be therefore more likely to have low level antibodies and higher risk of recurrence.

The number of potential predictors relative to the number of outcomes in our dataset was too large to use traditional approaches for building a multivariable logistic regression model. However, single predictor models did not allow us to control for confounding variables and estimate adjusted odds ratios. To overcome this limitation, we used a generalized elastic net regression to build a multivariable logistic regression model ${ }^{22,23}$ with the optimal number of predictors. The elastic net allows for fitting a multivariable model that includes a penalty for the number of predictors in the model so as to favor models in which several of the coefficients can be zero. In particular, we used a lasso penalty with leave-one-out cross validation to estimate the penalty parameter, and we report results using the penalty that gave the best cross-validated fit (smallest binomial deviance).

Following the approach of $\mathrm{Wu}$ and colleagues,${ }^{23}$ we report the odds ratio from a traditional multivariable logistic regression model using the predictors selected by the elastic net. We also give the leave-one-out index (LOO index), which is calculated using the likelihood ratio test to compare the model containing all predictors to a model with one predictor removed. The LOO indices are similar to a p-value in that smaller values indicate stronger statistical significance, but are not valid p-values because they ignore the complex model fitting used to select a final model. LOO indices are nonetheless useful in understanding the adjusted associations between the predictors retained in the final model. 


\section{Results}

Of 186 subjects in the cohort, 41(22\%) experienced an episode of rCDI. Of these 41 patients, $13(32 \%)$ had multiple recurrences, four of which had three episodes of rCDI.

In our study population, 53\% of the patients were male, and the median age was 7 years. Nearly $80 \%$ of the patients were Caucasian. Patient characteristics are summarized in Table 1. There were no statistically significant differences between the groups with respect to age, gender, or race. The majority ( $89 \%$ ) of the 186 patients had at least one identifiable comorbidity, with the most common being malignancy (40\%), stem cell transplant (15\%), gastroesophageal reflux disease (13\%), asthma (11\%), inflammatory bowel disease (11\%), and TPN dependence (11\%). (See Supplemental Digital Content Table 1 for detailed list). Patients with an episode of recurrence were more likely to carry a diagnosis of malignancy ( $71 \%$ of cases, $30 \%$ of controls, $\mathrm{p}<0.001)$ and less likely to carry a diagnosis of asthma ( $2 \%$ of cases and $14 \%$ of controls, $\mathrm{p}=0.043$ ). There was no statistically significant difference in the remainder of comorbidities. At the time of primary CDI, $149(80 \%)$ of the patients were inpatients and 37 (20\%) were outpatients. Based on the accepted definitions of CDI, 71(38\%) had HO-HCFA, 57 (31\%) had CA-CDAD, 47 (25\%) had CO-HCFA, and 11(6\%) had indeterminate disease.

All patients were required to have diarrhea as specified in the inclusion criteria. Twenty-one patients (11\%) had diarrhea alone, while 49\% also had fever, 50\% had abdominal pain, 55\% had tachycardia, $37 \%$ had vomiting, and $25 \%$ had grossly bloody stools. There were 10 CDI-related complications during the episode of primary CDI and these included: toxic megacolon $(\mathrm{N}=2)$, pseudomembranous colitis $(\mathrm{N}=3)$, the need for a surgical intervention $(\mathrm{N}=1)$, and the need for an ICU admission related to $\mathrm{CDI}(\mathrm{N}=4)$. No patients died in the cohort due to complications from CDI.

The majority of patients received metronidazole monotherapy for primary CDI (93\%). Of the remainder, ten patients received combination therapy with both metronidazole and oral vancomycin, and 3 patients received monotherapy with oral vancomycin.

We analyzed the 11 pre-specified predictors to determine their relationship with rCDI (Table 2). On univariable analysis, factors significantly associated with rCDI included the following: antibiotic, immunosuppressant, and acid blocker use during the 30 days prior to symptoms onset, the total number of antibiotics by class used at any time in the 30 day period, recent hospitalization, recent surgery, malignancy, and HO-HCFA.

Results obtained from the multivariable elastic net modeling procedure are presented in Table 3. Each of the 11 predictors in Table 2 were considered for potential inclusion, and a model with four predictors (malignancy, recent surgery, presence of a feeding tube, and the number of antibiotic exposures by classes) was selected as having the best cross-validated fit. For these predictors, the odds ratios from unadjusted, adjusted, and lasso-penalized logistic regressions (lasso OR) are presented. We also give the LOO index which describes the association of each predictor with recurrence after controlling for the other three predictors in the model. Malignancy is most strongly associated with the odds of recurrence with an odds ratio of 3.39 , and it has the smallest LOO index, indicating that it is the 
strongest adjusted association with recurrence. Feeding tube presence was associated with recurrence but had a higher LOO index indicating that it provided relatively little additional information on the odds of recurrence after accounting for the other predictors in the model. For all predictors, the lasso OR is closer to 1 than the adjusted odds ratio, which is expected because it adjusts for the complex model fitting process.

\section{Discussion}

For pediatric patients, we identified three main statistically significant predictors of rCDI, listed in order of importance: malignancy, recent surgery, and the number of antibiotic classes to which the patient was exposed. While antibiotic exposure is a shared risk factor among adults and children, the other predictors are novel risk factors in children. As opposed to previous adult studies, ${ }^{24,25}$ we did not identify an association between use of PPIs and rCDI in children. Also notable, the majority of patients in our study with CDI had an identifiable comorbidity. As opposed to adult studies where age $>65$ is an independent risk factor regardless of comorbidities, it appears that in children, the presence of a comorbidity may be the more important factor in risk of both primary and recurrent disease.

Malignancy was identified as the most important risk factor for rCDI in our pediatric population (adjusted $\mathrm{OR}=3.39,95 \% \mathrm{CI}=1.52-7.85$ ). Patients with malignancy have been identified as high risk for primary $C$. difficile disease. A recent study highlighted that pediatric oncology patients had the highest incidence of CDI as well as the highest increase in incidence over a 5 year period from 2006 through $2011 .{ }^{26}$ Our study is the first to demonstrate that these patients are also at the highest risk of recurrent disease.

An insufficient host immune response is likely an important factor in the development of rCDI. In adults, those with lower levels of serum IgM and IgG against toxin A are more likely to experience recurrence. ${ }^{13}$ Although patients with malignancy are more likely to have the other predictors analyzed by our study, our models demonstrated that malignancy remained an independent risk factor for rCDI. Of course, it is difficult to truly measure the burden of risk factors that may place pediatric patients with malignancy at heightened risk of rCDI. The degree of medication use and hospital exposures in these patients can be substantial. Although our statistical modeling will account for an additive effect of measured exposures, the cumulative effects may be difficult to fully delineate. It is notable however, that our study demonstrated low rates of both primary CDI and rCDI in children with cystic fibrosis despite the fact that these children also have high rates of antibiotic use and hospital exposures. Also notable, the 2012 study that demonstrated increased rates of CDI in children with malignancy did not demonstrate the same increases in CDI in hospitalized patients with inflammatory bowel disease or immunocompetent hospitalized children, again suggesting that there is something unique about the risk of CDI in children with malignancy outside of hospital exposures. ${ }^{26}$ Future studies should explore the degree and duration of medical encounters in patients with CDI to better address this important question.

Our study also identified a surgical procedure in the 60 days prior to onset of CDI symptoms as an independent risk factor for $\mathrm{rCDI}$ in children (adjusted $\mathrm{OR}=2.4,95 \% \mathrm{CI}=1.05-5.52$ ). Prior studies have demonstrated that patients undergoing bowel surgery are at higher risk of 
primary CDI ${ }^{27}$ but not rCDI. It has been hypothesized that the risk for primary CDI in these patients is due to the changes in the intestinal microbiome that accompany these procedures. ${ }^{27}$ However, in our pediatric patients who underwent surgery, just over half had the placement or removal of a central line. This type of surgery is less likely to directly impact the gut microbiome but does require the use of general anesthesia and narcotics. A mouse model demonstrated that animals exposed to morphine demonstrate significant suppression of intestinal mucus and disrupted intestinal epithelium. ${ }^{28}$ Although human studies have not yet confirmed these findings, the use of anesthetics and narcotics may lead to alterations in the gut microbiome, thus explaining the observed increased risk of rCDI in our patients.

Another plausible explanation for the risk of rCDI associated with surgical procedures in our patients may be due to the use of perioperative antibiotic prophylaxis (PAP). While we captured surgical procedures for 60 days prior to symptom onset, we limited antibiotic exposure to 30 days; as a result, PAP would not be captured in this cohort if surgery occurred between 31-60 days, and this is a limitation of our study. A recent study identified that $1.5 \%$ of patients who developed CDI during a $C$. difficile outbreak received PAP as their only antibiotic exposure. The authors suggested that when PAP is used to prevent only infrequent and minor infections, the risk of CDI needs to be taken into account. ${ }^{29}$

Antibiotics have long been implicated in the risk of primary CDI and more recently have been shown to increase the risk of rCDI in adults. ${ }^{11,12}$ This is likely due to the significant alterations in the intestinal microbiome that accompany antibiotic usage. Standard treatments of CDI are assumed to suppress $C$. difficile to a sufficient degree to allow recovery of the remaining microbiome to a "normal" state. ${ }^{30}$ It has been hypothesized that if the microbiome is deficient in its ability to recover, rCDI is likely. ${ }^{7}$ A 2008 study demonstrated that in adults with rCDI, the fecal microbiome was significantly decreased in phylotype richness compared with patients who experienced a single episode of CDI. ${ }^{30}$ Our study demonstrated that children with a higher number of antibiotic exposures by class (median of 3 classes in those with recurrence versus median of 1 class in controls) in the 30 days prior to symptom onset were more likely to suffer from rCDI (adjusted $\mathrm{OR}=1.33,95 \%$ $\mathrm{CI}=1.01-1.75)$. One patient who experienced $\mathrm{rCDI}$ in our cohort was exposed to eight antibiotic classes in the 30 days prior to symptom onset of primary CDI. These numbers not only highlight the risk of rCDI with increased antibiotic use, but they also highlight the need for antibiotic stewardship and the practice of evidence-based medicine in antibiotic use.

Our study has several important limitations. The retrospective nature of our study makes it possible that some episodes of recurrence were not captured. Our cohort exhibited a $22 \%$ recurrence rate, however, which is consistent with the rates identified in adults. ${ }^{5-7}$ There is also the possibility of ascertainment bias as we had to rely on information in the medical record to determine exposures. However, during the study period, MCJCHV was the only children's hospital and the only center with pediatric subspecialty care in a large geographic region, limiting the care that patients would receive at other institutions. As the majority of children in our cohort had associated comorbidities, it is more likely that their MCJCHV subspecialty physicians would have been made aware of any episodes of CDI diagnosed or treated by their primary care physician. As the electronic medical record used at MCJCHV 
includes all medical records and communications from outside physicians, this information would likely have been captured.

Our results are nearly two fold higher than those recently reported from a 9-year cohort study where $12 \%$ of children hospitalized with CDI had recurrent disease. ${ }^{15}$ Whether the rate differences in these two studies reflect better antibiotic stewardship at the other study site, differences in associated comorbidities, or incomplete capture of all the cases of rCDI is not clear. Our increased rate of recurrent disease may also reflect the ever increasing burden of the North American pulsed-field gel electrophoresis type 1 strain of $C$. difficile as this strain has been associated with increased rates of recurrent disease in adults. ${ }^{31}$ Receipt of concomitant antibiotics and community-associated CDI were independently associated with recurrent disease in the other study, but that study did not apply statistical modeling to account for the relatively small number of events. Both studies provide important new insights into $\mathrm{rCDI}$ in children.

Finally, our study was conducted at a single tertiary care hospital. Although our findings are applicable to other major children's hospital populations, these risk factors may not be applicable for outpatient children without comorbidities. Despite these limitations, our study provides some of the first insights into the important reasons why children experience rCDI, and it highlights the differences in risk factors between children and adults.

\section{Conclusions}

Although the burden of rCDI is substantial in adults, there have been very few studies evaluating the rates of recurrent disease in children and the associated risk factors in this population. We have identified three statistically significant risk factors for rCDI that, by order of importance, include malignancy, recent surgery and the number of antibiotic exposures by class. Malignancy and history of a recent surgery are novel risk factors that have not been implicated previously in recurrent disease in either adult or pediatric studies. As we approach the advent of newer therapies aimed at the prevention of $\mathrm{rCDI}$ and the potential for a $C$. difficile vaccine, identifying those patients at highest risk of $\mathrm{rCDI}$ is critical.

\section{Supplementary Material}

Refer to Web version on PubMed Central for supplementary material.

\section{Acknowledgments}

Grant Support: This study was partially supported by an NIH training grant, 5T32 DK007673-20 Peek (PI) NIH/ NIDDK Training in Gastroenterology and NIH/NCATS grant support UL1 TR000445 for REDCap.

\section{References}

1. Elliott B, Chang BJ, Golledge CL, et al. Clostridium difficile-associated diarrhoea. Intern Med J. 2007; 37:561-568. [PubMed: 17640189]

2. Zilberberg MD, Tillotson GS, McDonald C. Clostridium difficile infections among hospitalized children, United States, 1997-2006. Emerg Infect Dis. 2010; 16:604-609. [PubMed: 20350373] 
3. Khanna S, Baddour LM, Huskins WC, et al. The epidemiology of Clostridium difficile infection in children: a population-based study. Clin Infect Dis. 2013; 56:1401-1406. [PubMed: 23408679]

4. Kim J, Smathers SA, Prasad P, et al. Epidemiological features of Clostridium difficile-associated disease among inpatients at children's hospitals in the United States, 2001-2006. Pediatrics. 2008; 122:1266-1270. [PubMed: 19047244]

5. Kyne L, Kelly CP. Recurrent Clostridium difficile diarrhoea. Gut. 2001; 49:152-153. [PubMed: 11413124]

6. McFarland LV, Surawicz CM, Greenberg RN, et al. A randomized placebo-controlled trial of Saccharomyces boulardii in combination with standard antibiotics for Clostridium difficile disease. Jama. 1994; 271:1913-1918. [PubMed: 8201735]

7. Maroo S, Lamont JT. Recurrent clostridium difficile. Gastroenterology. 2006; 130:1311-1316. [PubMed: 16618421]

8. McFarland LV, Surawicz CM, Rubin M, et al. Recurrent Clostridium difficile disease: epidemiology and clinical characteristics. Infect Control Hosp Epidemiol. 1999; 20:43-50. [PubMed: 9927265]

9. McFarland LV, Elmer GW, Surawicz CM. Breaking the cycle: treatment strategies for 163 cases of recurrent Clostridium difficile disease. Am J Gastroenterol. 2002; 97:1769-1775. [PubMed: 12135033]

10. Garey KW, Sethi S, Yadav Y, et al. Meta-analysis to assess risk factors for recurrent Clostridium difficile infection. J Hosp Infect. 2008; 70:298-304. [PubMed: 18951661]

11. Johnson S. Recurrent Clostridium difficile infection: a review of risk factors, treatments, and outcomes. J Infect. 2009; 58:403-410. [PubMed: 19394704]

12. Eyre DW, Walker AS, Wyllie D, et al. Predictors of first recurrence of Clostridium difficile infection: implications for initial management. Clin Infect Dis. 2012; 55(Suppl 2):S77-S87. [PubMed: 22752869]

13. Kyne L, Warny M, Qamar A, et al. Association between antibody response to toxin A and protection against recurrent Clostridium difficile diarrhoea. Lancet. 2001; 357:189-193. [PubMed: 11213096]

14. D'Agostino RB Sr, Collins SH, Pencina KM, et al. Risk Estimation for Recurrent Clostridium difficile Infection Based on Clinical Factors. Clin Infect Dis. 2014; 58:1386-1393. [PubMed: 24599770]

15. Tschudin-Sutter S, Tamma PD, Milstone AM, et al. Predictors of First Recurrence of Clostridium difficile Infections in Children. Pediatr Infect Dis J. 2013

16. Jangi S, Lamont JT. Asymptomatic colonization by Clostridium difficile in infants: implications for disease in later life. J Pediatr Gastroenterol Nutr. 2010; 51:2-7. [PubMed: 20512057]

17. Cohen SH, Gerding DN, Johnson S, et al. Clinical practice guidelines for Clostridium difficile infection in adults: 2010 update by the society for healthcare epidemiology of America (SHEA) and the infectious diseases society of America (IDSA). Infect Control Hosp Epidemiol. 2010; 31:431-455. [PubMed: 20307191]

18. Obeid JS, McGraw CA, Minor BL, et al. Procurement of shared data instruments for Research Electronic Data Capture (REDCap). J Biomed Inform. 2013; 46:259-265. [PubMed: 23149159]

19. Zilberberg MD, Reske K, Olsen M, et al. Risk factors for recurrent Clostridium difficile infection (CDI) hospitalization among hospitalized patients with an initial CDI episode: a retrospective cohort study. BMC Infect Dis. 2014; 14:306. [PubMed: 24898123]

20. O'Keefe SJ. Tube feeding, the microbiota, and Clostridium difficile infection. World J Gastroenterol. 2010; 16:139-142. [PubMed: 20066732]

21. Lubbert C, Johann C, Kekule AS, et al. Immunosuppressive treatment as a risk factor for the occurrence of clostridium difficile infection (CDI). Z Gastroenterol. 2013; 51:1251-1258. [PubMed: 23696115]

22. Zou H, Hastie T. Regularization and Variable Selection via the Elastic Net. J. R. Statist. Soc. B. 2005; 67:301-320.

23. Wu TT, Chen YF, Hastie T, et al. Genome-wide association analysis by lasso penalized logistic regression. Bioinformatics. 2009; 25:714-721. [PubMed: 19176549]

24. Linsky A, Gupta K, Lawler EV, et al. Proton pump inhibitors and risk for recurrent Clostridium difficile infection. Arch Intern Med. 2010; 170:772-778. [PubMed: 20458084] 
25. Rotramel A, Poritz LS, Messaris E, et al. PPI therapy and albumin are better predictors of recurrent Clostridium difficile colitis than choice of antibiotics. J Gastrointest Surg. 2012; 16:2267-2273. [PubMed: 23007285]

26. Hojsak I, Ferenc T, Bojanic K, et al. Incidence of Clostridium difficile infection in children with inflammatory bowel disease compared to oncology and immunocompetent patients. Digestion. 2012; 86:6-11. [PubMed: 22688504]

27. Zerey M, Paton BL, Lincourt AE, et al. The burden of Clostridium difficile in surgical patients in the United States. Surg Infect (Larchmt). 2007; 8:557-566. [PubMed: 18171114]

28. Babrowski T, Holbrook C, Moss J, et al. Pseudomonas aeruginosa virulence expression is directly activated by morphine and is capable of causing lethal gut-derived sepsis in mice during chronic morphine administration. Ann Surg. 2012; 255:386-393. [PubMed: 21989372]

29. Carignan A, Allard C, Pepin J, et al. Risk of Clostridium difficile infection after perioperative antibacterial prophylaxis before and during an outbreak of infection due to a hypervirulent strain. Clin Infect Dis. 2008; 46:1838-1843. [PubMed: 18462108]

30. Chang JY, Antonopoulos DA, Kalra A, et al. Decreased diversity of the fecal Microbiome in recurrent Clostridium difficile-associated diarrhea. J Infect Dis. 2008; 197:435-438. [PubMed: 18199029]

31. Marsh JW, Arora R, Schlackman JL, et al. Association of relapse of Clostridium difficile disease with BI/NAP1/027. J Clin Microbiol. 2012; 50:4078-4082. [PubMed: 23052318] 


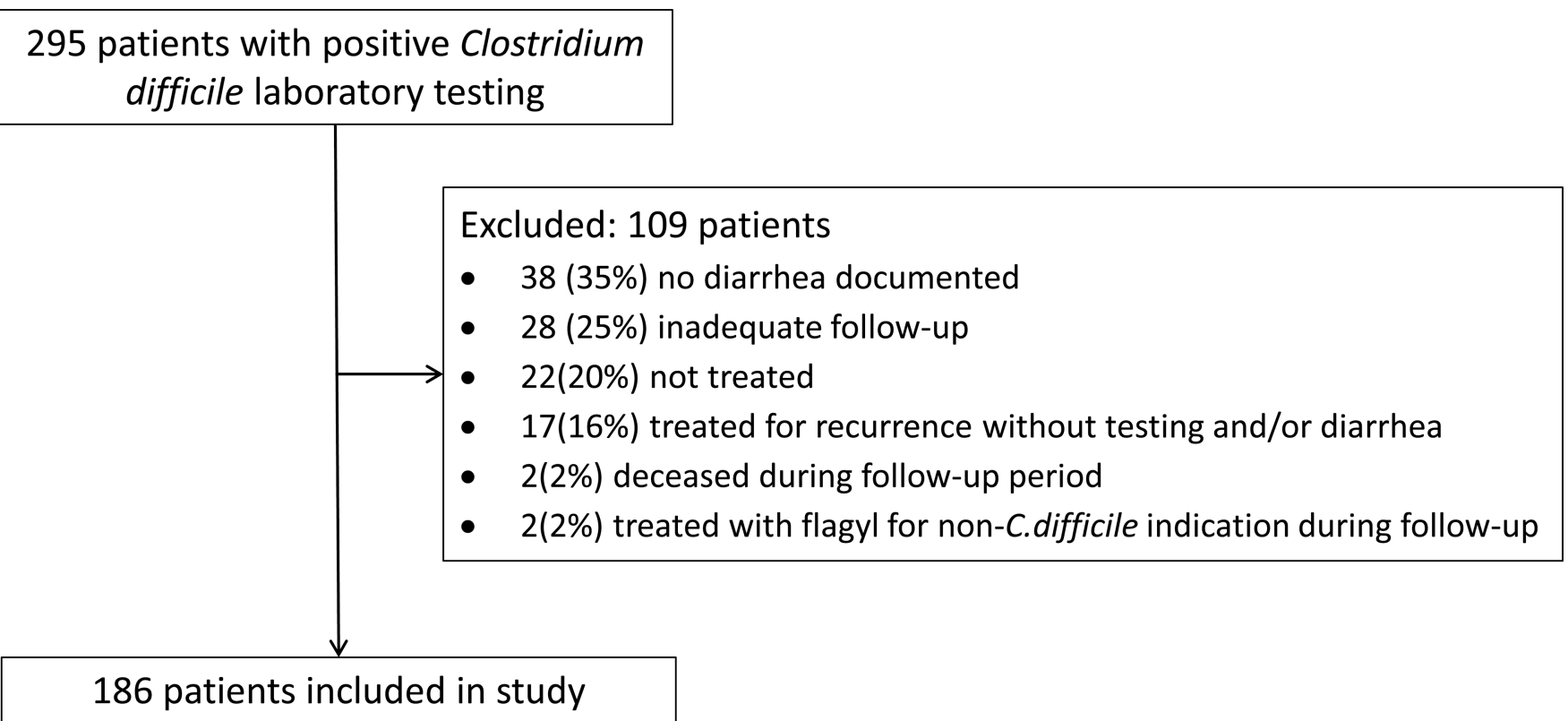

Figure 1.

Schematic of Patient Selection 
Table 1

Demographic Variables of Patients with and without Recurrent CDI

\begin{tabular}{|l|c|c|c|}
\hline & Recurrent CDI (N=41) & No Recurrence (N= 145) & P value \\
\hline Age (mean) & 7 & 7.9 & $0.36^{2}$ \\
Gender & & & $0.57^{1}$ \\
Boy & $20(49 \%)$ & $78(54 \%)$ & \\
Girl & $21(51 \%)$ & $67(46 \%)$ & \\
Race & & & $0.428^{1}$ \\
White & $33(80 \%)$ & $108(74 \%)$ & \\
Non-white and Unknown & $8(20 \%)$ & $37(26 \%)$ & \\
Location at time of CDI & & $113(78 \%)$ & $0.16^{1}$ \\
Inpatient & $36(88 \%)$ & $32(22 \%)$ & \\
Outpatient & $5(12 \%)$ & & $0.052^{1}$ \\
Type of CDI & & $49(34 \%)$ & \\
HO-HCFA & $22(54 \%)$ & $11(8 \%)$ & \\
CO-HCFA & $10(24 \%)$ & $48(33 \%)$ & \\
Indeterminate & $0(0 \%)$ & & \\
CA-CDAD & $9(22 \%)$ & & \\
\hline
\end{tabular}

CDI: Clostridium difficile infection

HO-HCFA: Healthcare facility-onset healthcare facility-associated

CO-HCFA: Community-onset healthcare facility-associated

CA-CDAD: Community associated Clostridium difficile associated disease

I Pearson test;

${ }^{2}$ Wilcoxon test 
Table 2

Considered Predictors of Recurrent Disease

\begin{tabular}{|l|c|c|c|}
\hline & Recurrent CDI (N=41) & No Recurrence (N=145) & P Value \\
\hline Malignancy & $29(71 \%)$ & $44(30 \%)$ & $<0.001^{1}$ \\
Presence of Feeding Tube & $17(41 \%)$ & $38(26 \%)$ & $0.059^{1}$ \\
Recent Hospitalization & $35(85 \%)$ & $100(69 \%)$ & $0.038^{1}$ \\
Recent Surgery & $26(63 \%)$ & $41(28 \%)$ & $<0.001^{1}$ \\
Antibiotic Use & $40(98 \%)$ & $113(78 \%)$ & $0.004^{1}$ \\
Number of Antibiotic Classes & 234 & 113 & $<0.001^{2}$ \\
Acid Blocker Use & $32(78 \%)$ & $84(58 \%)$ & $0.019^{1}$ \\
Proton Pump Inhibitor Use & $9(22 \%)$ & $37(26 \%)$ & $0.64^{1}$ \\
Immunosuppressant Use & $30(73 \%)$ & $63(43 \%)$ & $<0.001^{1}$ \\
Presence of Fever & $22(54 \%)$ & $72(50 \%)$ & $0.65^{1}$ \\
HO-HCFA & $22(54 \%)$ & $49(34 \%)$ & $0.021^{1}$ \\
\hline
\end{tabular}

HO-HCFA: Healthcare facility-onset healthcare facility-associated

abc represent the lower quartile $\mathrm{a}$, the median $\mathrm{b}$, and the upper quartile $\mathrm{c}$ for continuous variables

Pearson test;

${ }^{2}$ Wilcoxon test 
Table 3

Strongest Predictors of Recurrent Disease

\begin{tabular}{|l|c|c|c|c|}
\hline & Lasso OR & $\begin{array}{c}\text { Unadjusted OR } \\
(\mathbf{9 5 \%} \text { CI) }\end{array}$ & $\begin{array}{c}\text { Adjusted OR } \\
(\mathbf{9 5 \%} \text { CI) }\end{array}$ & LOO index \\
\hline Malignancy & 2.75 & $5.55(2.65-12.25)$ & $3.39(1.52-7.85)$ & 0.0028 \\
Recent Surgery & 1.96 & $4.40(2.14-9.32)$ & $2.40(1.05-5.52)$ & 0.0370 \\
Number of Antibiotic Classes & 1.27 & $1.66(1.32-2.13)$ & $1.33(1.01-1.75)$ & 0.0420 \\
Presence of Feeding Tube & 1.19 & $1.99(0.96-4.10)$ & $1.54(0.69-3.46)$ & 0.3000 \\
\hline
\end{tabular}

OR: Odds Ratio

LOO: Leave-one-out 